$\begin{array}{ll}\text { Italique } & \text { Italique } \\ \text { Poésie italienne de la Renaissance }\end{array}$

IV | 2001

Varia

\title{
Armi « in forma di trofeo » sul sepolcro di Clorinda (Gerusalemme Liberata XII 94-95)
}

\section{Guglielmo Gorni}

\section{(2) OpenEdition}

\section{Journals}

\section{Edizione digitale}

URL: http://journals.openedition.org/italique/180

DOI: 10.4000/italique.180

ISSN: 1663-4438

\section{Editore}

Librairie Droz

\section{Edizione cartacea}

Data di pubblicazione: 1 dicembre 2001

Paginazione: 103-121

ISBN: 2-600-00826-1

ISSN: 1423-3983

\section{Notizia bibliografica digitale}

Guglielmo Gorni, «Armi « in forma di trofeo » sul sepolcro di Clorinda (Gerusalemme Liberata XII 94-95 ) », Italique [Online], IV | 2001, online dal 06 octobre 2009, consultato il 01 mai 2019. URL : http:// journals.openedition.org/italique/180 ; DOI : 10.4000/italique.180 
GuGLIELMO GOR N I

A R M I « N FORMA D I T R O FE O»

S UL SEPOLCRO D I C L OR INDA

(GERUSALEMMELIBERATA XII 94-95) 


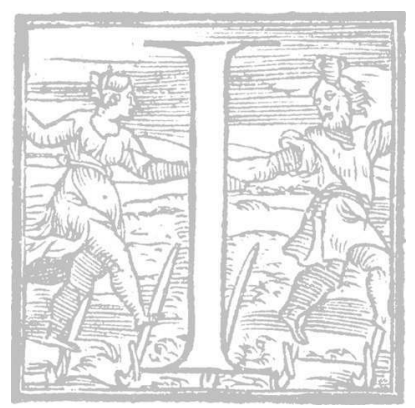

$N$ un saggio tassiano di qualche anno $f a$, avevo scritto incidentalmente che Tancredi, sepolte «le dilette membra» di Clorinda, aveva «appeso le sue armi presso il sepolcro dell'amata, in segno di omaggio e di rinuncia ormai a combattere»." Guido Baldassarri, recensendo con consenso quel mio scritto, ${ }^{2}$ mi obiettò senza addurre speciali argomenti, ma come a lume di buon senso, che le «arme» in questione, di cui il Tasso non precisa la proprietà, dovevano appartenere alla stessa Clorinda, non già al guerriero cristiano. Carteggiai a suo tempo con l'amico Baldassarri, ribadendo il mio punto di vista. Ma non trovai allora la prova certa, tratta dagli antichi, ${ }^{3}$ che armi disposte «in forma di trofeo» sul tumulo di un vinto a cui si vuole attribuire un postumo omaggio sono quelle del superstite afflitto. Oltre la generica impressione (anche se non infondata, come si vedra) che Tancredi volesse rendere alla donna uccisa l'onore delle armi - delle proprie armi, odiose a chi le portava per il sangue versato - non riuscivo ad andare. Il fatto è che, a norma della tradizione, l'invenzione del Tasso è una singolarità nel suo genere.

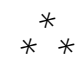

Riprendo le fila del discorso, non solo per corrobare la mia vecchia ipotesi, ma anche per mettere in luce la complessità del caso, che si riferisce a riti e costumi di cui si è perduto il senso e la memoria. ${ }^{4}$ Queste le ottave del celebre episodio:

Consolato ei si desta e si rimette

De' mendicanti a la discreta aita,

E intanto sepelir fa le dilette

Membra ch'informò già la nobil vita.

E se non fu di ricche pietre elette

La tomba e da man dedala scolpita,

Fu scelto almeno il sasso, e chi gli diede

Figura, quanto il tempo ivi concede.

Quivi da faci in lungo ordine accese

Con nobil pompa accompagnar la feo,

E le sue arme, a un nudo pin sospese,

Vi spiegò sovra in forma di trofeo.

(Liberata XII 94 e 95, I-4) 
Questi versi suonano cosi nella versione della Gerusalemme Conquistata:

Ei desto si consola, e 'nsin ch'aspette

Di medico gentil discreta aita,

Vuol che sepolte sian quelle dilette

Membra ch'informò già sì nobil vita.

E se non fu di ricche pietre elette

La bella tomba, e del suo amor scolpita,

Fu scelto almeno il sasso, e chi gli diede

La forma, quanto il tempo ivi concede.

Quivi da faci, in ordin lungo accese,

Con nobil pompa accompagnar la feo,

E le sue arme, a un nudo pin sospese,

Vi spiegò, quasi grande e bel trofeo.

(Conquistata XV I07 e 108, I-4) ${ }^{7}$

«Arme» di lui o «arme» di lei nel «trofeo» funebre? Il silenzio dei commentatori moderni, che magari indugiano a chiosare quanto nel testo è evidente, senza darsi cura di spiegare - poniamo - che cos'e un «trofeo», termine tecnico di un cerimoniale perento, è concorde al riguardo: o perché l'esegesi è sembrata ovvia (è forse quel che pensavano gli antichi), o perché non è stato avvertito il dilemma, o perché - nella generale reticenza degli interpreti - non ci si è voluti compromettere con una dichiarazione esplicita. Ma la questione non è affatto gratuita e val la pena di darsene pensiero.

$$
\text { ** }
$$

Poco o nulla si ricava da un trofeo d'armi, composto con intento metaforico di ardua decifrazione, che compare nelle stanze liriche Amor, contra costei che 'n treccia e 'n gonna, scritte nel Is 86 «ad istanza di Vincenzo Gonzaga, principe di Mantova». A quanto pare, qui le armi appese sono gli attributi della donna ostile e freddamente virtuosa, ma infine sconfitta. Nella seconda delle due ottave (nella panoplia si riconoscono l'egida col capo della Gorgone, l'asta di Minerva e l'arco di Diana, emblemi tutti di una castità ostile) si leggono questi versi, che presentano affinità lessicali coi precedenti del canto XII della Liberata:

Scudo ch'avvolge al capo atri serpenti

E d'elmo e di lorica il doppio incarco,

Grave faretra e strali ancor pungenti, 
E l'asta d'una diva e d'altra l'arco

Amor sospende a le future genti,

Né di pietà né di piacer mai parco,

A ciò ch'insieme un sol trofeo dimostri

Due vittorie e cento armi e mille mostri.

$\left(\right.$ Rime 446) ${ }^{8}$

Una prova e silentio a sostegno dell'ipotesi che le armi sul sepolcro siano quelle di Clorinda si può forse addurre a norma di un'altra ottava della Liberata, se - quando Tancredi torna a indossare «elmo» e «lorica» - non si accenna a una nuova armatura che egli porti:

Era il prence Tancredi intanto sorto

A sepelir la sua diletta amica,

E benché in volto sia languido e smorto

E mal atto a portar elmo e lorica,

Nulla di men, poi che 'l bisogno ha scorto,

Ei non ricusa il rischio o la fatica,

Ché 'l cor vivace il suo vigor trasfonde

$\mathrm{Al}$ corpo sì che par ch'esso n'abbonde.

(Liberata XIII 32)

Sennonché non si dà qui una speciale vestizione del guerriero, e $i$ termini sono troppo generici per trarne le conclusioni che si sono postulate. ${ }^{9}$ Né insisto sul fatto che, a rigore, le vere armi di Clorinda non erano accessibili a Tancredi: dove avrebbe mai potuto recuperarle per appenderle al sepolcro, considerato che quelle che la donna portava nel duello notturno erano armi ingannevoli, «ruginose e nere»?

E le due ottave del terzo libro che narrano il funerale di Dudone, a cui è presente Goffredo, mescolano ancor più i fili della storia invece di sdipanarne la matassa:

Sorse a pari co 'l sole, ed egli stesso

Seguir la pompa funeral poi volle.

A Dudon d'odorifero cipresso

Composto hanno un sepolcro a piè d'un colle,

Non lunge a gli steccati; e sovra ad esso

Un'altissima palma i rami estolle.

Or qui fu posto, e i sacerdoti intanto

Quiete a l'alma gli pregàr co 'l canto. 
Quinci e quindi fra i rami erano appese Insegne e prigioniere arme diverse, Già da lui tolte in più felici imprese A le genti di Siria ed a le perse.

De la corazza sua, de l'altro arnese, In mezzo il grosso tronco si coperse. «Qui» vi fu scritto poi «giace Dudone: Onorate l'altissimo campione».

(Liberata III 72-73)

Nella Conquistata, dove Dudone è detto Guidone, la seconda ottava suona così:

Quinci e quindi fra' rami eran sospese
Spoglie di foggia e di color diverso,
Già da lui tolte in più felici imprese
Al guerier di Bithinia, al Siro, al Perso.
La sua propria lorica, e l'altro arnese
Il gran tronco vestì, di sangue asperso.
«Quivi» fu scritto poi «giace Guidone:
Onorate l'altissimo campione».

$\left(\right.$ Conquistata IV 79) ${ }^{10}$

Ci sono dunque armi di nemici vinti, sui rami; ma sul «tronco» della palma prendono posto quelle di lui Dudone, talché la situazione resta ancipite. Scipio Gentili, a proposito dell'ottava di Liberata III 72 citata più sopra, approvava la scelta dell'ora mattutina, non quella dell'albero:

Sente quel costume antico di sepelire gli morti nell'Aurora. Per il che Platone nel suo Commune comanda che il corpo del Censore si porti a sepellire nel far del sole, e che intorno al sepolcro molti alberi vi si piantino. Et intendi degli alberi selvaggi et infruttiferi, perché tali dice lo Scholiaste di Apollonio che si solevano piantare intorno al sepolchro degli Heroi. Il che noto non fuor di proposito o per incidenza, ma perché il Tasso fa che Dudone sia posto sotto una palma, la quale in quegli paesi produce frutti suavissimi. E certo quel buon Ciro lasciò nella morte sua di essere sepolto in terra, ove ancora morto potesse giovare gli huomini, con dare nutrimento alle piante et alle sterpi. Sì come recita Senofonte nella Istitutione di esso, lib. ultimo. ${ }^{11}$

E Francesco Birago cerca di dar ragione di quella che, a parer suo, è un'incongruenza del Tasso, 
Viene Guidone honorato non solo d'un gran sepolcro, ma ancho d'un altier Trofeo conforme allo Stile degli antichi Romani, li quali in questo modo gli huomini, che stato [sic] valorosi erano, havevano, non solo perché honesto è, ma perché è utile alla Città, per incorar li giovani ad opre virtuose, et forse il Poeta in questo luogo esser ripreso potrebbe, perché orna il Trofeo dell'armi proprie di Guidone contro l'antica usanza, essendo che sopra gli arbori, come da Virgilio nel principio dell'undecimo si raccoglie, si ponevano se non l'armi tolte a' nimici, et sopra li sepolcri le proprie, come dal luogo di Virgilio di sopra notato alla stanza 78, appare: «At pius Aeneas ingenti mole sepulchrum / Imponit suaque arma viro remumque tubamque». ${ }^{12}$ Si risponde, che li Trofei erano o a loro stessi, o a' morti inalzati, se a sé stesso alcuni drizzava un Trofeo, come fece Enea nel principio dell'undecimo dell'armi nimiche solo pomposo lo rendeva; ma s'a' morti, delle proprie armi, et delle nimiche l'ornava.

A ben vedere, il caso di Clorinda non è lo stesso, perché qui le armi altrui sono antiche prede di Dudone, "Insegne e prigioniere arme diverse, / Già da lui tolte in più felici imprese» (Liberata III 72, 2-3). Un privilegio di vincitore, che Clorinda non potrebbe rivendicare a sé. E vana sarebbe, al riguardo, una sorta di galanteria postuma da parte di Tancredi, che infranga le convenzioni. A meno che Tancredi, vincitore, si volesse, sul sepolcro di lei, vinto. É questa l'ipotesi di lettura che a me piace più di ogni altra.

$E$ si veda infine quanto il Birago scrive riguardo a Conquistata XVII 58-59, e in particolare sui versi 6-8 dell'ottava 58, che attestano la tracotanza di Ormondo nel progettare un trofeo delle armi di Goffredo, dato per vinto: «... premio altro non chiede / Se d'alzar un bel trofeo de l'arme / In Babilonia, e sotto un breve carme».

Tolto da Omero nel settimo dell'́liade. Voleva Ormondo ergere un Arco trionfale, et ornarlo delle spoglie di Goffredo; percioché, nelle Città si fabricavano gli Archi. Trofei sono le spoglie, et vittoriose prede fatte de' nimici, et anticamente, come nota Servio ${ }^{13}$ nel i lib. di Virg. s'inalzavano li trofei de' nemici sconfitti, e si trionfava de gli uccisi, et prima furono drizzati li trofei sovra arbori, de' quali tagliati i rami, gli appicavano le spoglie de' nemici dopo si fecero di marmi ne' monti et luoghi alti, et ultimamente nelle Città sovra alcuni Archi, per questo fabricati. Che le spoglie nel trofeo erano sopra gli arbori posti [sic], ce lo mostra Virg. nell'undecimo quando dice: «Mezenti ducis exuvias, tibi, magne, tropaeum» ${ }^{14}$ et quel, che segue, et di questo si è scritto nel libro quarto stanza 79.

St. 59 Perché memoria ad ogni età trapassi.

Rende la cagione perché si dirizzavano li trofei. ${ }^{15}$ 
Tale passo nella Liberata suonava cosi:

Parla il duce a colui: - Dunque securo

Sei così tu di dar morte a Goffredo? -

Risponde quegli: - Io sonne, e 'n corte giuro

Non tornar mai se vincitor non riedo.

Preverrò ben color che meco furo

Al congiurare; e premio altro non chiedo

Se non ch'io possa un bel trofeo de l'armi

Drizzar nel Cairo, e sottopor tai carmi:

"Queste arme in guerra al capitan francese,

Distruggitor de l'Asia, Ormondo trasse

Quando gli trasse l'alma, e le sospese

Perché memoria ad ogni età ne passe».

- Non fia - l'altro dicea - che 'l re cortese

L'opera grande inonorata lasse:

Ben ei darà ciò che per te si chiede,

Ma congiunta l'avrai d'alta mercede.

(Liberata XIX 63-64)

Non bontà di cavalieri antiqui sul sepolcro della donna: semmai una tragica rinuncia e un solenne Nunc dimittis. Si può inoltre notare che il «sospese» del passo su Clorinda è più vicino all'«appese» (che diverrà senz'altro «sospese» nella Conquistata), riferito alle armi altrui, che al «si coperse», riferito a quelle del defunto, del passo su Dudone. E forse se ne può concludere che se Tancredi avesse voluto disporre sul pino le armi di Clorinda, avrebbe scelto una posizione diversa: ne avrebbe cioè ricoperto il sepolcro o 'rivestito' il tronco, anziché 'sospenderle' ai rami. Si può pensare che l'ellittico brano del canto XII sottintenda in qualche misura le minuziose informazioni fornite dal III. Resta almeno appurato che la posizione delle une e delle altre è distinta: le armi nemiche tra i rami, quelle dell'eroe sul tronco, che ne è rivestito quasi antropomorficamente, come se rappresentasse il corpo del defunto. ${ }^{16}$ Anche la funzione dei due gruppi di armi è diversa: quelle del morto tramandano ai posteri la sua identità; quelle dei nemici sono la prova manifesta della sua prestanza in guerra.

Si ponga mente infine che Tancredi «sospese»le armi «a un nudo pin»: perché mai «nudo»? O perché semplicemente spogliato dei rami, ma non se ne vede il senso e la ragione. $O$ «nudo» perché non rivestito di armi (al contrario dell'albero di Dudone, che ne è coperto, e della quercia di Mezenzio, induta di esse, come si vedrà). Ė da preferire la seconda ipotesi, anche perché $i$ verbi 
«sospese» e «spiegò soura» non si possono riferire che ai rami o alla chioma dell'albero. E insomma pare di dover ribadire che il vincitore Tancredi è come un vinto di Clorinda, vuol essere postumamente una sua preda, consacrata dal lutto. Il Tasso, è ben vero, non speculò su questa situazione singolarissima, che pure sembra cosi congeniale alla sua invenzione concettosa e manierista: segno, forse, di un imbarazzo teorico grave, paralizzante per il poeta.

$$
\text { * * }
$$

A sostegno della mia interpretazione si può citare un sonetto tardo, ultimo di cinque componimenti, un'ottava e quattro sonetti (Rime I430-I434), scritti in morte (2 marzo I589) del cardinal Alessandro Farnese. Il sonetto inscena una prosopopea di Roma dolente, certo memore della personificazione della città nella Farsaglia di Lucano (I I85-92). Nella prima quartina, lillustre matrona si recide la chioma, coronata o composta di lugubri frondi, cipressi e ginepri (figurazione tipicamente barocca, insieme umana e vegetale); l'appende al sepolcro del porporato, o anzi la brucia, in segno di cordoglio:

«Questa mia di cipresso e di ginebro

Squallida chioma io tronco, e qui l'appendo,»

Disse Roma nel lutto «anzi l'accendo

Con mille faci, e 'l mio dolor celebro».

$(\text { Rime } \mathrm{I} 434)^{17}$

Roma dunque orna la tomba del cardinal Farnese con qualcosa di suo, manifestando la propria afflizione con un dono che ̀̀ propriamente una mutilazione di sé.

$E$ anche Goffredo nell'ottava finale della Liberata lascia in voto al Sepolcro di Cristo le proprie armi: come ancor oggi, nei santuari piu frequentati, si appendono ex voto e oggetti per grazia ricevuta.

Così vince Goffredo, ed a lui tanto

Avanza ancor de la diurna luce

Ch'a la città già liberata, al santo

Ostel di Cristo i vincitor conduce.

Né pur deposto il sanguinoso manto,

Viene al tempio con gli altri il sommo duce;

E qui l'arme sospende, e qui devoto

Il gran Sepolcro adora e scioglie il voto.

$\left(\right.$ Liberata XX I44) ${ }^{18}$ 
Alla luce di questi passi sembra di poter concludere che a Clorinda morta è reso l'onore delle armi di chi l'ba uccisa: armi che d'ora innanzi, per disperazione e per ammenda, il preterintenzionale uccisore rinuncia a portare. Un'esegesi che ha, mi pare, $i$ connotati dell'evidenza e il sostegno - come si è visto - della tradizione, che induce ad appendere armi altrui sull' albero adibito a trofeo sul sepolcro.

$$
\text { * * }
$$

Sennonché bisogna ammettere che le cose non sono così semplici. Anzitutto è da dire che l'esempio di Goffredo che si è appena prodotto vien meno, se si passa dalla Liberata alla Conquistata. Nel rifacimento del poema il Tasso ba cambiato le modalità della cerimonia: sono votate al Sepolcro non già le armi del capitano, come in una specie di rinuncia che abbia luogo alla fine delle ostilità, bensi le spoglie dei nemici, trofeo di gloria riservato a un dio fatto nomo, che assume qui gli attributi di re degli eserciti: ${ }^{19}$

Quasi in trionfo par che spieghi e mostri

Il vincitor de l'onorate imprese

E disarmati i carri e gl'indi mostri,

E l'alte insegne già squarciate e prese;

E con macchine eccelse, antenne e rostri,

Et auree spoglie, e vario e ricco arnese;

E vòte le faretre, e rotti gli archi

E di ferro i prigioni avinti e carchi.

$[\cdots]$

Dove Sion, pendendo al lucid'orto,

Copre ritonda mole a' primi raggi,

Giacque il gran Re, ch'in croce affisso e morto

Trionfò de la morte e de gli oltraggi.

Qui venerâr la tomba, ond'ei risorto

Poscia a' suoi fidi apparve alti messaggi;

E 'l duce, di pietà sublime essempio,

Donò le spoglie e sciolse i voti al tempio.

(Conquistata XXIV I 34 e I 37)

Identica era stata, nell' ultimo canto della Liberata, la promessa di Tisaferno, che aveva votato al suo dio Macone le armi del nemico Rinaldo. Voto pio e legittimo, anche se vano, perché sarà Rinaldo ad avere il sopravvento su di lui. ${ }^{20}$ E significativo che questo episodio di Tisaferne (tale il suo nome nella 
Conquistata) sia soppresso nella revisione del poema, talché nel XXIV canto, che pur ne racconta la morte per mano di Goffredo, non ne resta traccia.

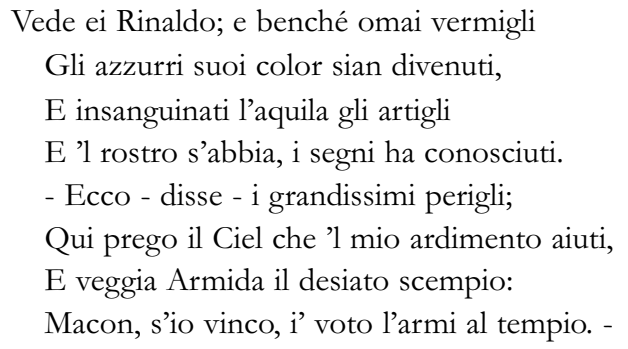

(Liberata XX I I 3 )

La volontà ultima del Tasso epico, in materia di trionfi d'armi, trofei e pompe funebri, è attestata dalle ottave 107-30 della Conquistata, dove tra l'altro si dà conto della sepoltura d'Argante e di altri, e dove in genere si mette la sordina a ogni celebrazione troppo francamente pagana in Terra Santa (basti citare, in bocca di Goffredo, "Troppo, abi, troppo di male oggi s'è visto, / E fatto preda abbiam d'argento e d'oro», Liberata XXIII IIs, 5-б). Tutte queste sono ottave nuove, che nella Liberata non figuravano, e che introducono un'apologia implicita di vari riti funebri, tra cui la cristiana inumazione dei cadaveri. Ecco l'ultima ottava del canto:

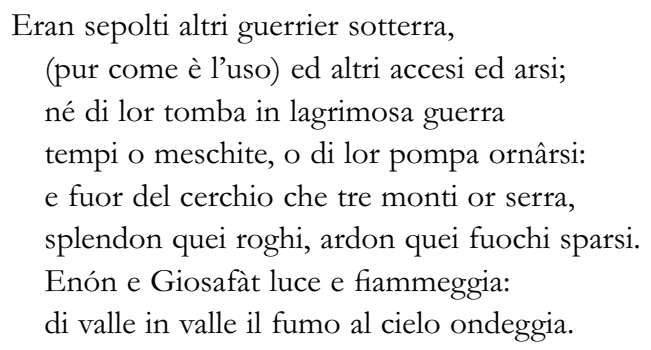

(Conquistata XXIII I 30)

Per il sontuoso e scenografico Tasso, specie dell'ultima maniera, non è pensabile che la tomba di un illustre estinto resti disadorna e priva di «trofeo». Rivolgendosi al vincitore di Lepanto, don Giovanni d'Austria, il Tasso ba scritto: «Ma tu, che lui [il Trace, cioè il Turco] fugasti, in quale arene / O 'n qual libera terra or sei sepolto? / Qual trofeo s'erge in tua memoria o tempio?》 (Rime 774, I2-I4), concetto ripreso in un altro sonetto indiriz?ato alla città di Napoli, che ospita la tomba del famoso ammiraglio cristiano, decorata, come è costume, di armi, insegne e bandiere prese ai vinti: «Vi 
mostro a dito alto trofeo / Di spoglie tolte al barbaro rapace» (Rime I42I, $7-8)$.

Ma la storia di Tancredi è tutt'altra. C'è l'onore reso all'estinta, e c'è la rinuncia di lui a combattere più oltre, un appendere al chiodo le proprie armi sciagurate una volta per tutte. Il controverso episodio deve avere imbarazzato lo stesso autore, espertissimo di cose cavalleresche, come sa bene, nei Promessi Sposi, il conte Attilio. ${ }^{21}$ Passando dalla Liberata alla Conquistata, il Tasso si è dato cura di rettificare il comportamento di Goffredo, riportandolo al cerimoniale consueto: con una perdita di efficacia narrativa, a mio parere, ma con perfetto galateo. Viceversa ha lasciato che la pompa funebre di Clorinda restasse nell'ombra della sua congenita ambiguità, proprio per non poter dare una norma canonica a quanto ba fatto Tancredi.

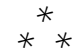

In questo caso intricato - come Virgilio insegna, e con buona pace di Dudone o Guidone, che peraltro non è responsabile del postumo omaggio che gli è reso - le spoglie dei vinti non spettano alle creature, ma si offrono piuttosto a Dio. A questa stregua, armi «in forma di trofeo» sul sepolcro di Clorinda non potrebbero essere di nemici vinti da lei o da Tancredi. Arredare la tomba con tale suppellettile sarebbe stata un'empietà, quella stessa censurata in Mezenzio, ${ }^{22}$ che prima di affrontare Enea in duello aveva promesso le armi di lui al proprio figlio Lauso:

«Dextra mihi deus et telum, quod missile libro, Nunc adsint! voveo praedonis corpore raptis Indutum spoliis ipsum te, Lause, tropaeum Aeneae».

Le armi di un vinto non si possono ostentare irritualmente. Non è forse il malfatato balteo di Pallante indossato da Turno a perdere Turno? L'uso disinvolto, implicitamente sacrilego, di quella reliquia suscita l'ira di Enea, vincendone le perplessità; e nell'epilogo del poema lo spinge a vendicare Pallante, uccidendo l'uccisore di lui. Dopo la supplica di Turno,

Stetit acer in armis

Aeneas volvens oculos dextramque repressit;

Et iam iamque magis cunctantem flectere sermo

Coeperat, infelix umero cum apparuit alto

Balteus et notis fulserunt cingula bullis 
Pallantis pueri, victum quem volnere Turnus

Straverat atque umeris inimicum insigne gerebat.

(Aen. XII 938-44)

Il rapido fulgore del balteo di Pallante sull' armatura del re Etrusco condanna irreparabilmente chi lo porta, facendone una vittima espiatoria dovuta ai mani del giovane ucciso:

«Tune hinc spoliis indute meorum

Eripiare mihi? Pallas, te hoc volnere Pallas

Immolat et poenam scelerato ex sanguine sumit».

(Aen. XII 947-49)

Oppure le armi dei vinti, e in particolare gli scudi, vanno posti sul rogo, in un rito d'immolazione al dio Vulcano: come attesta Evandro, che da giovane aveva compiuto questo gesto:

«O mihi praeteritos referat si Iuppiter annos,

Qualis eram, cum primam aciem Praeneste sub ipsa

Stravi scutorumque incendi victor acervos».

$(\text { Aen. VIII } 560-62)^{24}$

In un autore assillato da scrupoli come il Tasso, tutto ciò pesava molto sullinvenzione, fin quasi a spegnerla.

$$
*^{*} *
$$

Quali dunque le auctoritates e i modelli impliciti dell'invenzione del Tasso, in tanta incertezza di segni? Il genere letterario da addurre a modello del gesto di Tancredi, non è il poema epico, eroico o cavalleresco nella sua lunga storia, ma piuttosto - a me sembra - l'epigramma votivo (ed eventualmente quello funebre), di cui è raccolta vasta messe nell'Antologia Palatina. ${ }^{25}$ Penso specialmente agli epigrammi che si leggono nel sesto libro dell'Antologia, $i$ quali - senza che si possa enucleare un modello preciso per il Tasso - dicono l'offerta degli strumenti dell'arte, fatta da un cultore che se ne stacca per ragioni d'età o altre, a una divinità protettrice. Vecchi pescatori o cacciatori che appendono le reti al simulacro di Posidone, di Ermes o alle Ninfe; agricoltori che consacrano i loro attrezzi dismessi a Demetra, Pan o Priapo; altri ancora le spole ad Atena, lancia e scudo ad Ares, l'arco a Febo, le penne alle Muse, e cosi via. Più raramente si votano alla divinità le spoglie di nemici vinti: come, poniamo, a Zeus, nell'epigramma VI 332. Ė vero che la rinuncia di Tancredi 
non è assoluta, ma solo relativa, legata a una condizione di lutto (dopo tutto, il nostro eroe non abbandona il campo di Gerusalemme, e invece gli epigrammi che si sono menzionati segnano una totale cessazione di attività da parte di chi depone per sempre i propri arnesi). Ma nella rinuncia s'intravede, mi pare, una mortificazione votiva, si manifesta una condizione non aliena dalla tradizione epigrammatica.

E un altro precedente illustre per la discrezione sottile ed esigente del Tasso ̀̀ poi quello della settima egloga di Virgilio, dove Corydon prega le Ninfe che gli concedano un canto pari almeno a quello di Codro, altrimenti egli appenderà il proprio strumento musicale a un pino, albero sacro a Pan (è appena il caso di rammentare che un «nudo pin» ̀̀ eretto sulla tomba di Clorinda):

«Nimphae, noster amor, Libethrides, aut mihi carmen, Quale meo Codro, concedite (proxima Phoebi Versibus ille facit), aut, si non possumus omnes, Hic arguta sacra pendebit fistula pinu».

(Buc. VII 21-24)

Agli occhi del Tasso - anche se non resta alcuna testimonianza in proposito l'inserzione di una maniera caratteristica dell'epigramma votivo (la consegna delle proprie armi) o della poesia bucolica a un certo punto dovette parere incongrua, per coerenza di genere. Per Goffredo si poteva rimediare, cambiando le regole del gioco, ma che fare nel caso di Clorinda, a costo di sfidare l'empietà?

Non potevano riservarsi a lei spoglie di nemici, che - come si è detto - spettano solo a Dio: Tancredi, a quel punto, sarebbe stato in sospetto d'idolatria. D'altra parte appendere le armi della donna (ammesso e non concesso che fossero disponibili a Tancredi) al sepolcro di lei sarebbe stata una procedura inadeguata, se l'intento era quello di tributare all'amata estinta un omaggio straordinario: che onore additizio poteva mai venirle? Il Tasso avrebbe potuto sopprimere l'episodio nella Conquistata, e però non l'ha fatto: ha lasciato l'ambiguità di un gesto non previsto dal cerimoniale, senza darne più precisa informazione. Per una volta tanto, il poema regolare cedeva alle ragioni della poesia. Forse è un'interpretazione che può apparire azzardata rispetto alle premesse teoriche dell'autore. Ma è tempo di compromettersi, e non vedo spiegazione più persuasiva di questa.

$$
\text { ** }
$$

L'appassionante caso delle armi deposte sul sepolcro di Clorinda, di chi siano mai, sembra dunque avviato a soluzione. Non dico che passi fin d'ora in 
giudicato, ma in grazia della presente inchiesta, la questione, non meno curiosa che dotta, ha fatto un bel passo avanti. Se sono «in forma di trofeo», o atte a formare «quasi grande e bel trofeo», tali armi non potranno a nessun patto esser quelle di Clorinda: lo vietano tutti gli autori, e più chiaramente di tutti il Birago. O si tratterà di un trofeo «pomposo», di sole armi nemiche, o di un trofeo misto di armi proprie e altrui, come pure è lecito (ma il Tasso dice semplicemente, anche se ambiguamente, "le sue armi», e non autorizza tale interpretazione). E se dunque le armi altrui son quelle di Tancredi, significativamente s'innova qui la tradizione, e si fa di un vincitore disperato un vinto volontario.

Discorrendo di questi fatti, chi scrive si è sentito, a un certo punto, collega stretto di don Ferrante, ammiratore e complice, come si sa, di quel rinomato gentiluomo che era Francesco Birago; $;^{26}$ la cosa inquieta, perché anche in uno studio recente su altra materia mi era toccato di scoprire insospettate affinità elettive con quel saggio d'altri tempi, oggi tanto screditato. ${ }^{27} \dot{E}$ che non c'è scampo. E questo il prezzo da pagare alla memoria storica, che a noi tutti - si dice - sta a cuore. A costo di riabilitare la cultura barocca, gusti e maniere di quell'età «sudicia e sfarzosa», $X$ (Manzoni) e convertire quanto è stato oggetto di ironia e dileggio in ricerca serissima. E a patto forse, come si cerca di fare qui, di avvertire il cortese lettore, esserne coscienti come operatori e saperne sorridere a tempo e luogo. Certo, chi si fa beffe della filologia avrà ora qualche freccia in più al suo arco, anche se la questione affrontata riguarda sì, inevitabilmente, il galateo delle armi sul sepolcro, ma deve fare $i$ conti anche con una nuova definizione del personaggio di Tancredi, o con l'invenzione funebre del Tasso nelle sue complesse implicazioni teoriche e religiose, e tocca insomma un nodo cruciale del poema. Per schivare la Cariddi della chiacchiera a vuoto, è inevitabile che si cada nella Scilla di una connivenza colpevole coi propri testi. ${ }^{28}$

Guglielmo Gorni 
I. Cfr. G. Gorni, Il "gran sepolcro» di Gerusalemme. Sacro e finzione del sacro nel Tasso, «Compar(a)ison». An International Journal of Comparative Literature, 2 (1994), pp. 77-92, numero monografico dedicato a Jerusalem. Visions, Phantasies and Transpositions of the Holy City, a cura di Aminadav Dykman e Michael Jakob. La citazione è da p. 87 .

2. G. Baldassarri, «Studi Tassiani», XLIII (1995), pp. I4I-42. In questa nota Baldassarri pone anche le premesse per l'esegesi di un altro passo funebre rimasto inesplicato, l'ottava 32 del canto VIII sul miracoloso ritrovamento del corpo di Sveno nella carneficina dei crociati danesi. Ma in proposito si veda quel che pensava Giulio Guastavini, Discorsi et Annotationi sovra la Gierusalemme liberata di Torquato Tasso, Pavia, Heredi Bartoli, i 592, p. I6o: «E par che senta quello, che dice Suetonio nella vita di Cesare, che ne' primi giuochi che fece Ottavio in onor di lui, si vide per alcuni dì una stella crinita, la quale fu creduta l'anima di lui ricevuto in cielo».

3. Oltre ai repertori e ai dizionari di ordinaria consultazione, si veda almeno il capitolo Burial and the Cult of the Dead in W. Burket, Greek Religion, translated by J. Raffan, Cambridge (Ma), Harvard University Press, I985 (ed. originale Stuttgart, Kohlhammer, 1977), pp. $190-94$.

4. Da recuperare soprattutto alla luce di Francesco Birago, Dicbiarationi, et Avertimenti Poetici, Istorici, Politici, Cavallereschi et Morali nella Gerusalemme Conquistata del signor Torquato Tasso. Con la Tavola delle cose più notabili, che in essi si contengono, Milano, Benedetto Somasco, i6i6, dei citati Discorsi del Guastavini, e di Scipio Gentili, Annotationi sopra la Gierusalemme Liberata di Torquato Tasso, Leida [sic] I 586 . Le chiose degli ultimi due sono raccolte largamente nella stampa La Gierusalemme Liberata di Torquato Tasso, Con le Figure di Bernardo Castello, E le Annotationi di Scipio Gentili e di Giulio Guastavini, Genova, Bertoli, I 590, ristampata più volte tra Sei e Settecento. Taluno scrive Scipione Gentili, ma il nome è proprio Scipio, come anche rivela il frontespizio della traduzione in latino dei primi due canti della Liberata, Scipir Gentilis Solymeidos libri duo priores, la cui princeps è del i 584 .

5. Su cui verte un mio saggio, Il chiasmo di Clorinda, «Colloquium Helveticum», 2 (1985), pp. 8I-96, stampato anche col titolo redazionale Il combattimento di Tancredi e Clorinda: un'interpretazione tassiana, «Atti dell'Ateneo di Scienze, Lettere ed Arti, Bergamo», XLV (1984-1985), pp. 397-410.

6. Cito la Liberata dal testo a cura di Lanfranco Caretti, Milano, Mondadori, I957, con qualche ritocco d'interpunzione e nell'uso delle maiuscole iniziali di verso (che si sono restituite in ogni testo antico citato): certe parole importanti per la dimostrazione sono state tacitamente stampate in corsivo. Noto che, a quanto si ricava dagli autori, l'albero deputato alle ostensioni di panoplie è in genere la quercia. Ma si rammenti, per quel che può valere per lo scrupolosissimo Tasso, che Zerbino raccolse le armi sparse di Orlando, ormai divenuto furioso, proprio su un pino: «Quivi Zerbin tutte raguna l'arme / $\mathrm{E}$ ne fa come un bel trofeo su 'n pino; / E volendo vietar che non se n'arme / Cavallier paesan né peregrino, / Scrive nel verde ceppo in breve carme: / 'Armatura d'Orlando paladino'; / Come volesse dir: Nessun la muova, / che star non possa con Orlando a prova» (Furioso XXIV 57), circostanza ribadita a XXXI 43, 3-8. Una specie di Noli me tangere: *Rolandi sum. Il pino è supporto di oggetti votivi o apotropaici già presso Virgilio, come in «Hic arguta sacra pendebit fistula pinu» (Egl. VII 24) e «Oscilla ex alta suspendunt mollia pinu» (Georg. II 389). Altro è l'albero votivo nel quale si conficca irreparabilmente l'asta di Enea nell'ultimo duello con Turno, narrato in Aen. XII 766-90: «Forte sacer Fauno foliis oleaster amaris / Hic steterat, nautis olim venerabile lignum, / Servati ex undis ubi figere dona solebant / Laurenti divo et votas suspendere vestis» (sono i vv. 766-69).

7. Cito la Conquistata dall'editio princeps di Roma, Guglielmo Facciotti, I593, nell'esemplare della Biblioteca Civica «Angelo Mai» di Bergamo, alla quale appartiene ogni altra opera 
pertinente al Tasso, non senza tener conto della sua ristampa moderna, Torquato Tasso, Gerusalemme Conquistata, a cura di Luigi Bonfigli, 2 volumi, Bari, Laterza, 1934, ritoccando in genere l'interpunzione. Si noti che nel testo seriore del poema si dice «quasi grande e bel trofeo», dove quel quasi, con valore restrittivo, è un abile espediente dell'autore per mettersi al riparo da future contestazioni e per uscire una buona volta dall'intricato caso in cui s'era messo.

8. Cfr. Torquato Tasso, Opere, a cura di Bruno Maier, I. Aminta. Amor fuggitivo. Intermedi. Rime, Milano, Rizzoli, 1963, pp. 484-85.

9. Non sarebbe la prima volta che il Tasso, in materia di armi, incorre in una svista. Già Galileo notava che quando Clorinda indossa armi «ruginose e nere» (Liberata XII I 8, 4), non ha bisogno di deporre quelle «inteste / D'argento» (vv. I-2), perché gliele ha già rubate Erminia ( $«$ Se bene ho a memoria, non occorreva deporle, perché già erano state portate via da Erminia»: cfr. Galileo Galilei, Considerazioni al Tasso, in Scritti letterari, a cura di Alberto Chiari, Firenze, Le Monnier, 1970, pp. 594-95).

ı. Qui vi o Quivi non possono essere varianti d'autore, e l'una o l'altra forma andrà rettificata.

i i. Cfr. Scipio Gentili, Annotationi, p. 5 I.

I 2. Cfr. Aen. VI 232-33, sulle esequie di Miseno, in cui le armi del morto (cfr. v. 217, dove la pira è decorata con «fulgentibus armis», le sue) non sono arse sulla pira, come anche ci si poteva aspettare, ma deposte sul sepolcro.

13. Cfr. Servii Grammatici qui fervntvr in Vergilii Carmina Commentarii. II. Aeneidos librorvm VI-XII Commentarii, recensvit Georgius Thilo, Hildesheim, Olms, 196I [Retrographischer Nachdruck der Ausgabe Leipzig I 883-I 884], p. 477. [Commento a XI 6, constituit tumulo]: «in colle, quia tropaea non figebantur nisi in eminentioribus locis: Sallustius de Pompeio ait devictis Hispanis tropaea in Pyrenaei iugis constituit. ex quo more in urbibus tropaea figuntur arcubus exaedificatis. ideo autem nunc tropaeum ponit Aeneas, quia nondum plenam est victoriam consecutus, sed occiso Mezentio fugavit exercitum: plenae enim victoriae, ut supra $<\mathrm{X} 775$ : «indutum spoliis ipsum te, Lause, tropaeum» $>$ diximus, triumphus debetur. et persolvit vota [cfr. le ultime parole del poema, «e scioglie il voto»] vel propter tanti ducis interitum, vel quia fas erat etiam de primitiis belli sacrificare».

I4. Cfr. Aen. XI 7 (esametro citato nuovamente, col contesto, anche nella nota 23). Ho adattato alla lezione moderna, per comodità, le citazioni dai classici.

i s. Francesco Birago, Dichiarationi, p. 43 I.

I6. Il passo in questione sembra vicino a Aen. XI 5 sgg. citato più oltre nella nota 23, per il verbo «induit» e il particolare dei rami tagliati, «decisis undique ramis». L'immagine dell'albero 'rivestito' delle armi, sostituto simbolico del corpo del defunto, può rammentare la selva incantata del canto XIII della Liberata. Basti dire le parole di Ismeno ai demoni: «Prendete in guardia questa selva, e queste / Piante che numerate a voi consegno. / Come il corpo è de l'alma albergo e veste, / Così d'alcun di voi sia ciascun legno» (XIII 8, I-4), e soprattutto della falsa Clorinda a Tancredi: «Tu dal corpo che meco e per me visse, / Felice albergo già, mi discacciasti: / Perché il misero tronco, a cui m'affisse / Il mio duro destino, anco mi guasti? / Dopo la morte gli aversari tuoi, / Crudel, ne' lor sepolcri offender vuoi?») (XIII $42,3-8)$.

17. Cfr. T. TAsso, Opere, a cura di Bruno Maier, II. Rime, Rinaldo, Il Re Torrismondo, Milano, Rizzoli, 1964, pp. I 57-58. 
i 8. Giulio Guastavini, Discorsi, p. 344: «S’adempie quanto, ragionando a prencipi dell'oste nel primo canto, havea detto Goffredo essere suo disegno in que' versi: 'Né sia chi neghi al peregrin devoto / D'adorar la gran tomba, e sciòrre il voto'» [Liberata I 23, 7-8: anche la rima corrispondente devoto è la stessa]. Peraltro, a parte quanto si dice nei versi citati, il voto di Goffredo non risulta essere ritualmente formulato in nessun punto del poema.

19. Cfr. Torquato Tasso, Giudicio sovra la Gerusalemme riformata, a cura di Claudio Gigante, Roma, Salerno, 2000, II, $\ 73$, p. I 2 I: «in questa [Gerusalemme] nuovamente riformata il termine de la favola e l'ultimo confine è non la regia del Soldano o 'l castello da l'armi barbare occupato, ma 'l sacro tempio de la resurrezione ed il sepolcro di Cristo, con la sospensione delle spoglie ostili e con l'adempimento del voto di quel invittissimo principe de' principi e duce de' duci cristiani».

20. Giulio Guastavini nelle sue citate note al poema (Genova, Bartoli, I 590 ) menziona opportunamente il voto emesso da Pallante prima del duello con Aleso: «Da nunc, Thybri pater, ferro, quod missile libro, / Fortunam atque viam duri per pectus Halesi; / Haec arma exuviasque viri tua quercus habebit» (Aen. X 42 I-24).

21. Cito dal Fermo e Lucia, dove il conte Attilio, per la parte che ci interessa (I v), si chiama conte Orazio. «Le torno a dire, Signor Podestà riverito, che l'autorità del Tasso non serve al suo assunto, che è anzi è contro di lei, - riprese ad urlare il Conte Orazio: - perchè quel grand'uomo che conosceva tutte le regole e tutti i puntigli della cavalleria più soprafina ha fatto che il messo di Argante prima di esporre la sfida ai cavalieri cristiani, domandi licenza a Goffredo ...): cfr. Fermo e Lucia. Prima composizione del 1821-1823, in Tutte le opere di Alessandro Manzoni, a cura di Alberto Chiari e Fausto Ghisalberti, Milano, Mondadori, Volume II. Tomo III, I964³ p. 84.

22. Si tenga conto anche del lungo brano che il Giudicio dedica all'empietà di Mezenzio (II, \222-26, pp. 174-177), per cui l'editore allega i Saturnali di Macrobio (p. 175, nota 317).

23. Cito da Virgilio, Eneide, a cura di Ettore Paratore, traduzione di Luca Canali, volumi I-VI, Fondazione Lorenzo Valla, Milano, Mondadori, VI, 1983. Il commentatore ad locum, pp. 297-98, cita il parere di Dumézil, secondo cui Virgilio, «facendo pronunciare a Mezenzio il proposito sacrilego (come annota Servio) d'offrire le spoglie di Enea al figlio Lauso anziché a Giove come spoglie opime, ha adombrato la notizia relativa ai Vinalia [per cui cfr. soprattutto Ovidio, Fasti IV 879-900], trasferendola al culto delle spoglie opime; Enea risponderebbe a Mezenzio in XI 4-1 I, quando offre agli dei le spoglie del vinto guerriero etrusco. Anche quest'altra ipotesi dello studioso francese ci sembra dubbia». La diffidenza di Paratore sembra eccessiva: tra l'empio Mezenzio («contemptor ... deum»), che non ha altro dio che la sua destra, e il pius Aeneas, che riserverà a Marte le spoglie del vinto Etrusco, vige indubbiamente un continuo confronto a distanza. Il passo a cui si fa riferimento dice, scorciando: «Ingentem quercum decisis undique ramis / Constituit tumulo fulgentiaque induit arma, / Mezenti ducis exuvias, tibi, magne, tropaeum, / Bellipotens» (Aen. XI 5-8). La censura di Servio sul voto pronunciato di Mezenzio (Aen. X 775) è questa: «Sciendum sane Lauso propriam ideo nihil profuisse pietatem, quod in eum pater mente sacrilega munera transferebat deorum et ei occisorum spolia devovebat» (cfr. Servio, Commentarii, II, p. 467).

24. Servio, Commentarii, II, p. 279, chiosa ad locum: «hoc traxit de historia: Tarquinius enim Priscus victis Sabinis in honorem Vulcani eorum arma succendit: quem postea secuti sunt ceteri».

25. Cfr. Antologia Palatina, a cura di Filippo Maria Pontani, Torino, Einaudi, voll. I-IV, più volte ristampata. Il sesto libro di Epigrammi votivi, che comprende 358 pezzi con testo 
originale a fronte, è nel volume I (Libri I-VI, I 980²), pp. 285-469, il settimo di Epigrammi tombali, che ne comprende 748, è nel volume II (Libri VII-VIII, I979), pp. 3-373. Si tenga conto naturalmente che al tempo del Tasso l'Antologia era nota, anche a stampa, nella sua versione Planudea, di consistenza e ordinamento diversi dalla Palatina, menzionata qui solo per comodità di citazione in assenza di più puntuali indicazioni di fonti, che - a mio parere - sarebbe fuori luogo produrre. Sull' Antologia (Anthología diafóron epigrammáton) come uno dei possibili modelli della Giuntina di rime antiche del I 527 , anche per la formula di specificazione di diversi antichi autori toscani in dieci libri raccolte che segue il titolo Sonetti e canzoni, si veda, di chi scrive, Di qua e di là dal dolce stile, in Il nodo della lingua e il Verbo d'Amore. Studi su Dante e altri duecentisti, Firenze, Olschki, 198I, pp. 229-30. Delle molte stampe cinquecentesche dell'Antologia ho sott'occhio il Florilegium diversorum epigrammatum in septem libros [...] Magno epigrammatum numero et duobus indicibus auctum ( I 566), edito da Henri Estienne, nell'esemplare della Bibliothèque Publique et Universitaire di Ginevra: in questa stampa si veda, poniamo, a p. 4I 3 l'epigramma votivo di Antologia Palatina VI 25 . Non mi è riuscito invece di trovare il citato VI 332.

26. Nel Fermo e Lucia (III Ix), dove don Ferrante si chiama don Valeriano, si legge: «i Consigli e i Discorsi cavallereschi di Francesco Birago erano forse i libri più logori della sua biblioteca. Anzi Don Valeriano affermava, o faceva intendere spesso che quel grand'uomo non aveva sdegnato di consultarlo su certi casi più rematici; e parlando talvolta di quelle opere con quella venerazione che meritavano, e che per verità ottenevano da tutti, Don Valeriano aggiungeva misteriosamente: 'Basta: ho messo anch'io un zampino in quei libri'» (cfr. Fermo e Lucia, p. 498).

27. Alludo al mio contributo Ancora sui «paragrafi» della Vita Nova, «Rivista di Letteratura italiana», XIII (1995), 3, pp. 537-62 (passim).

28. La sostanza del presente saggio è stata discussa tempo fa (e ancora di recente) con Matteo Residori, allievo della Normale di Pisa, allora perfezionando a Ginevra. Mi è grato confessare il molto che devo ai suoi suggerimenti e rendergli insomma l'onore delle armi. 